\title{
CARACTERES MORFOLÓGICOS DE BOLBITIS SERRATIFOLIA (DRYOPTERIDACEAE) DE ARGENTINA
}

\author{
MARÍA DEL CARMEN LAVALLE ${ }^{1}$ \& MÓNICA RODRÍGUEZ
}

\begin{abstract}
Summary: Lavalle, M. C. \& M. Rodríguez. 2014. Morphological characters of Bolbitis serratifolia (Dryopteridaceae) from Argentina. Bonplandia 23(1): 33-42.

The study of this species was based on the morphological characters of the sporophyte with the goal of contributing new data to the understanding of the genus. It was compare to another genus of Dryopteridaceae and different diagnostic characters were proved. Architecture of fronds, morphology of the indument of blade and axis, epidermal patterns of pinna, stomata and spores were under consideration for this study. Dimorphic leaves, imparipinnate, anastomosing veins with free included excurrent veinlets, stem provided with scales, glabrous blades, and trilete spores are morphological characters that allow identifying this species.
\end{abstract}

Key words: Dryopteridaceae, Bolbitis serratifolia, Argentina, sporophyte, morphology.

Resumen: Lavalle, M. C. \& M. Rodríguez. 2014. Caracteres morfológicos de Bolbitis serratifolia (Dryopteridaceae) de Argentina. Bonplandia 23(1): 33-42.

Se estudió Bolbitis serratifolia sobre la base de rasgos morfológicos del esporófito, con el fin de aportar nuevos datos al conocimiento del género; se lo comparó con otro género de la misma familia y se pusieron a prueba distintos caracteres diagnósticos. Se trabajó sobre arquitectura foliar, venación, indumento, modelo epidérmico de las pinnas, estomas y esporas. El dimorfismo foliar, las frondes imparipinnadas, la venación reticulada con venilla excurrente, los ejes escamosos, las láminas glabras y las esporas triletes; son caracteres morfológicos que permiten identificar esta especie.

Palabras clave: Dryopteridaceae, Bolbitis serratifolia, Argentina, esporófito, morfología.

\section{Introducción}

El presente trabajo es continuación de contribuciones previas de las autoras sobre las Dryopteridaceae Herter de Argentina (Lavalle \& Rodríguez, 2009; 2010). Bolbitis Schott es un género pantropical que está ubicado en esta familia sobre la base de estudios morfológicos (Tryon \& Tryon, 1982; Mickel, 1991; 1999) y filogenéticos moleculares (Smith et al., 2006; Moran et al., 2007;
Ranker \& Haufler, 2008; Christenhusz et al., 2011). Este género está incluido en la tribu Bolbitideae Pic.-Ser (Tryon \& Tryon, 1982) y en la subfamilia Elaphoglossoideae (Pic. Serm.) Crabbe, Jermy \& Mickel (Christenhusz et al., 2011). Fue ubicado también en la familia Lomariopsidaceae Alston (Pichi Sermolli, 1969; de la Sota, 1973, 1977; Kaur, 1974; Kramer, 1990; Moran, 1995; Ponce, 1996; Ponce \& de la Sota, 2008).

La familia Dryopteridaceae está ampliamente

${ }^{1}$ Facultad de Ciencias Naturales y Museo. Universidad Nacional de La Plata. mdelclavalle@hotmail.com; moroca57@ gmail.com 


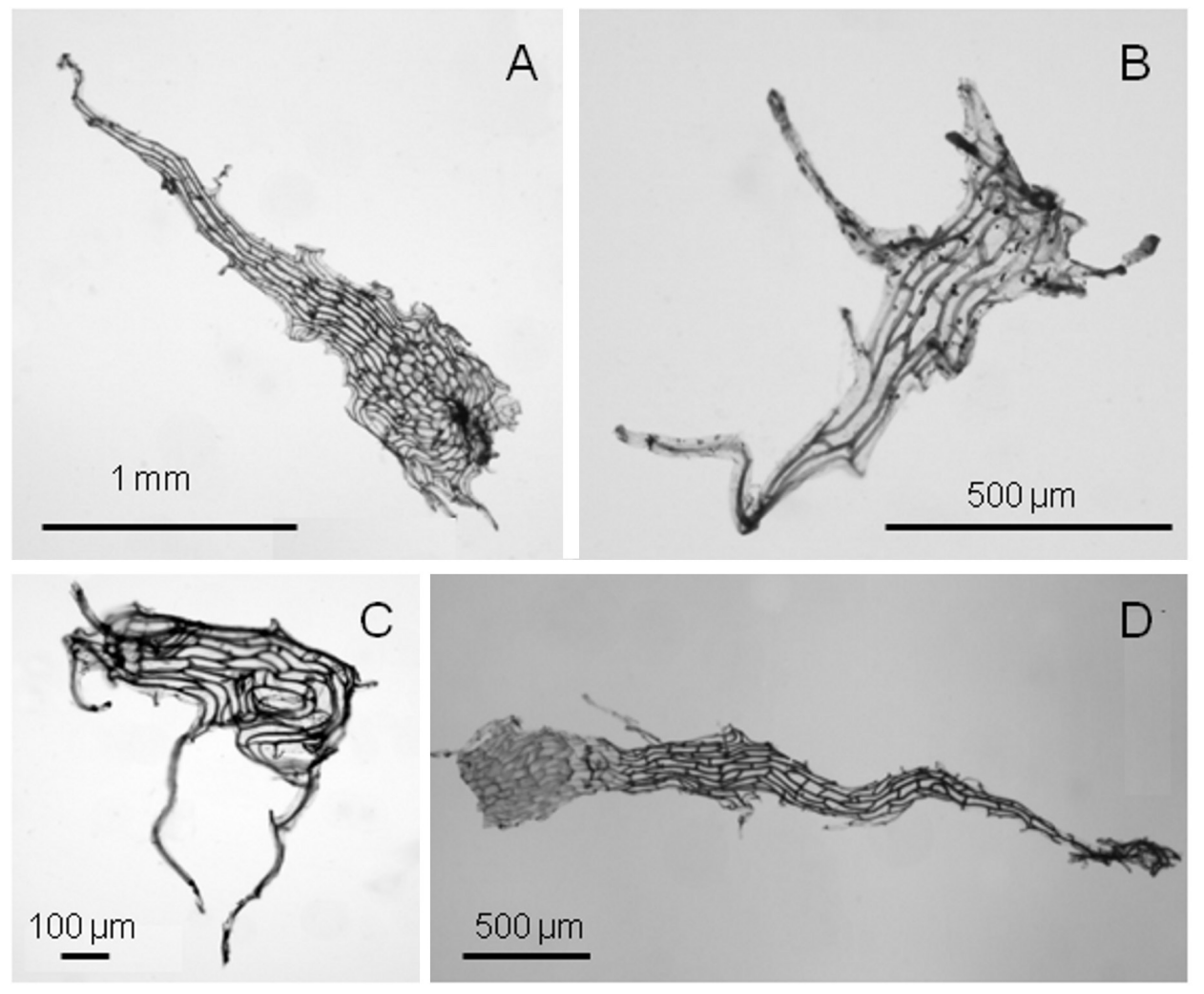

Fig. 1. Indumento en Bolbitis serratifolia. A: Escama del rizoma. B: Escama del estípite. C: Escama de la costa. D: Escama del raquis. (A, C: Iudica \& Ramadori 372; B, D: Palaci 622).

distribuida, el número de géneros difiere entre los autores, especialmente con respecto a los representantes del este de Asia, donde se ubica el centro de diversidad (Tryon \& Tryon, 1982). Estos autores incluyen más de 50 géneros, 30 de ellos americanos; Li \& Lu (2006) consideran cerca de 35 géneros; mientras que para Smith et al. (2006) esta familia contiene 1700 especies.

Existe una estrecha relación entre Bolbitis y Elaphoglossum, sustentada mediante datos de secuencia de ADN cloroplastidial por Moran et al. (2007).

El número de especies incluido en el género Bolbitis ha variado de acuerdo con los distintos criterios de los autores y en el transcurso del tiempo: 85 especies (de la Sota, 1973), 44 especies y 13 híbridos (Hennipman, 1977), 45 especies (Tryon \& Tryon, 1982; Mickel, 1991; Moran, 1995; Mickel \& Smith, 2004).

La especie $B$. serratifolia neotropical está íntimamente relacionada o es posiblemente coespecífica con la especie africana $B$. acrostichoides (Afzel. ex Sw.) Ching (Hennipman, 1977; Moran et al., 2010a).

El área de distribución de Bolbitis abarca, en el neotrópico, a México, Centro América, Antillas y Sudamérica; y en el paleotrópico, se extiende en África, sur de India, Sri Lanka, Malasia, norte y este de China, sur de Japón, noroeste de Australia y, a lo largo de las islas del Pacífico hasta Tahití y Rapa.

Bolbitis serratifolia aparece nombrada en catálogos (Ponce, 1996, Ponce \& de la Sota, 2008) y en el contexto de floras pteridológicas regionales (de la Sota, 1977; Smith, 1981; Mickel, 1991; Moran, 1995; Mickel \& Smith, 2004). Hay escasos trabajos morfológicos que analicen los distintos aspectos del esporófito tales como: patrones de venación, perisporio y rizomas (Hennipman, 1977); o características de las esporas (Tryon \& Lugardon, 1991; Moran et al., 2010b). Es una especie terrestre, presenta esporófitos que alcanzan aproximadamente unos 90 a $140 \mathrm{~cm}$ de altura. Se encuentra en el 


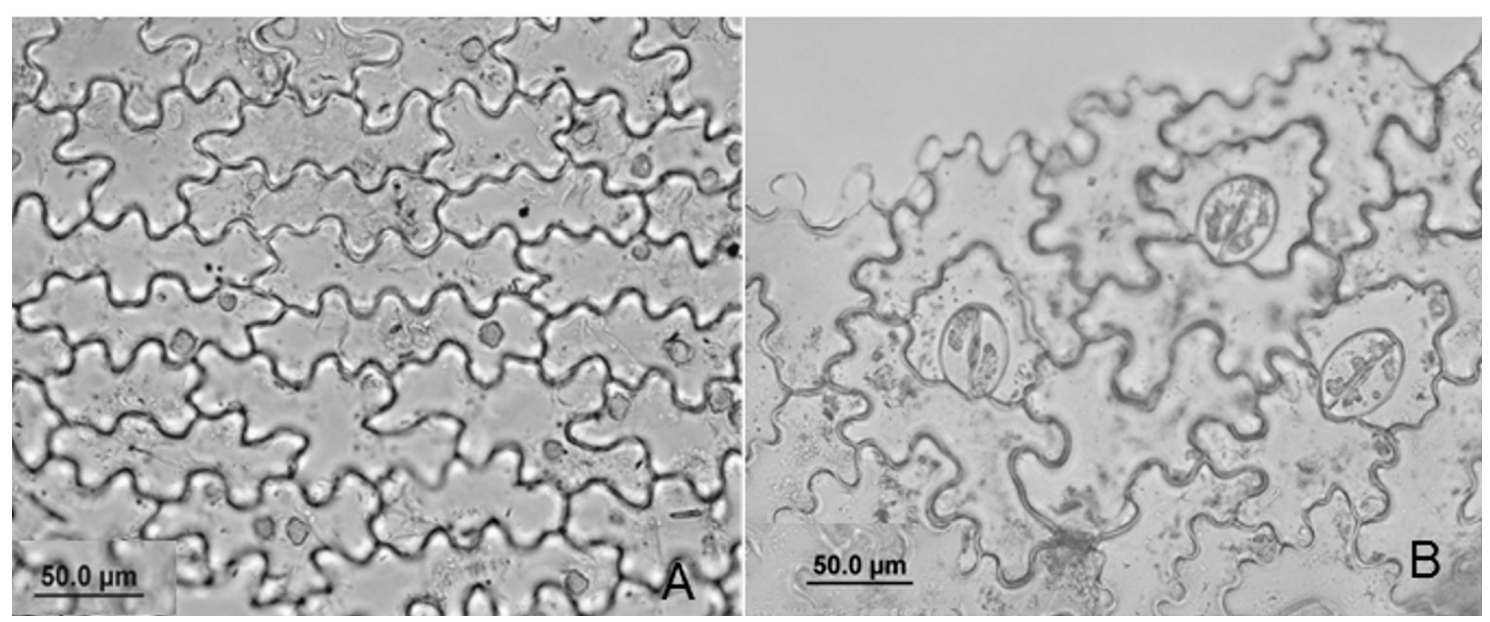

Fig. 2. Modelos epidérmicos y estomas en Bolbitis serratifolia. A: Superficie adaxial. B: Superficie abaxial.

sur de México, Nicaragua, Costa Rica, Panamá, Colombia, Venezuela, Guayanas, Surinam, Ecuador, Perú, Bolivia, Brasil, Paraguay y noroeste de Argentina. Crece desde selvas húmedas hasta selvas estacionalmente secas, en selvas de neblina, en selvas bajas de montaña, en sotobosques de selva basal en hábitats sombríos, y a lo largo de cursos de agua, en cañones, en nacientes de arroyos, donde está a menudo sobre piedra arenisca, limo u otras rocas; desde el nivel del mar hasta los $1800 \mathrm{~m}$, siendo más frecuente alrededor de los $1000 \mathrm{~m}$.

El objetivo de este trabajo es caracterizar a $B$. serratifolia analizando los rasgos morfológicos y su diversidad, aportando datos que contribuyan a mejorar el conocimiento de la especie. Se han analizado distintos caracteres del esporófito tales como tipo de rizoma; longitud de los estípites; contorno, textura, tamaño, margen, forma de ápice y base de las pinnas; patrones de venación; morfología y distribución del indumento; modelos epidérmicos de las láminas y ornamentación de los exosporios y perisporios de las esporas.

\section{Materiales y Métodos}

Se consultaron los herbarios BA, LIL, LP y SI (abreviaturas según Holmgren et al., 1990), los ejemplares analizados se citan a continuación:

ARGENTINA. Jujuy: Dep. Ledesma:
Calilegua, El Cafetal, 25-VI-1945, Capurro 217 (BA). Ledesma, 14-IX-1991, Guaglianone et al. 2638 (SI); 7-XII-1986, Iudica \& Ramadori 372 (SI). Salta: Dep. Orán: Aguas Blancas, Arroyo las Moras, 18-VII-1986, Palaci 622 (LP); Aguas Blancas, 21-VII-1945, Capurro 335 (BA); Camino a Finca Yakulica, a $33 \mathrm{~km}$ de Aguas Blancas próximo Río Pescado, 11-IX-1971, Legname \& Cuezzo 8439 C (LIL, LP); Río Pescado, pasando la finca de Yaculika $8 \mathrm{~km}$, camino a la Quebrada El Arrayasal, 25-X-1970, Vervoorst \& Cuezzo $7805 C$ (LIL); El Angosto del río Pescado (Finca Yakulica), 23-IX-1974, Meneses y Vervoorst 23 (LIL); Río San Andrés, 13-VII-1945, Capurro 296 (BA). Dep. General San Martín: confluencia del río Itahú y Bermejo, VII-1967, Zainer 61650 (BA); 15-XI-1986, Charpin \& Eskuche 20409AC (SI). Yariguarenda, Arroyo Las Piletas, 6-VIII-1945, Capurro 380 (BA); Yariguarenda, 3-VII-1944, Schulz \& Varela 5179 (LIL).

Para analizar el indumento se ablandaron los ejes en una solución de éter monobutílico del etilenglicol diluido 1:1 en agua, durante una semana a temperatura ambiente. El indumento de las láminas se observó en material diafanizado.

Para estudiar los modelos epidérmicos y los estomas de la lámina, se rehidrataron trozos de pinna, con agua y unas gotas de detergente. Se aclararon con hidróxido de sodio 5\% acuoso, se blanquearon con hipoclorito de sodio comercial diluido 1:1 en agua y se colorearon con TBO 
(azul de toluidina) 1\% acuoso. El radio celular (RC) indica la relación largo/ancho de las células.

Para estudiar la arquitectura foliar, se dibujaron al natural, esporófitos constituidos por frondas fértiles y estériles que luego se redujeron. Las ilustraciones y medidas corresponden a rasgos adultos de pinnas medianas.

Para el estudio de esporas con MEB se tomaron esporangios cerrados de las pinnas, se presionaron entre dos portaobjetos y las esporas se colocaron sobre porta-muestras de metal $(\mathrm{Cu}-$ $\mathrm{Zn}$ ) con cinta adhesiva doble faz. Las esporas, sin tratamiento previo, fueron recubiertas con oro bajo vacío con un metalizador Jeol JFC1100. Las fotografías se tomaron en el Servicio de Microscopía Electrónica de Barrido de la Facultad de Ciencias Naturales y Museo de La Plata, con un microscopio Jeol JSM-T100.

Las ilustraciones se realizaron con un microscopio Wild M-20 y un microscopio estereoscópico Nikon SM Z 1000, ambos equipados con cámara de dibujo. Las fotografías fueron tomadas con una cámara Nikon Optical Zoom 6,3 Mp; $10 \times$ Zoom y con microscopio Olympus BX51 equipado con cámara digital Olympus DP71.

La terminología relacionada con el indumento y las esporas es la propuesta por Lellinger (2002), la utilizada para describir las células epidérmicas, la de Rolleri \& Deferrari (1986) y para los tipos estomáticos adultos, la de Prabhakar (2004).

\section{Resultados}

\section{Indumento}

Los rizomas están tapizados por escamas persistentes, adpresas, concolores o bicolores, canela con la parte distal castaña, subclatradas a clatradas, basifijas o peltadas, lanceadas a deltoides, de márgenes subenteros con escasas y cortas lacinias distribuidas en todo el contorno, con bases cordadas, ápices cortamente acuminados y textura papirácea. Las escamas están constituidas por células cortas en la base y largas en el resto de la superficie (Fig. 1A).

Los estípites llevan escamas persistentes, patentes, concolores, canela, contrastantes con el color del estípite, peltadas, de contorno similar a las del rizoma, de márgenes subenteros con 2-5 prolongaciones aisladas largas, con bases cordadas y ápices acuminados (Fig. 1B). Las escamas están próximas entre sí, esparcidas en toda la longitud del estípite, pero con mayor densidad en la parte basal del mismo.

Los raquis (Fig. 1D) y las costas (Fig. 1C) presentan escamas de dos tipos, unas similares a las del rizoma y otras a las del estípite. Los epifilos e hipofilos son glabros.

\section{Modelos epidérmicos}

Las pinnas presentan modelo sinuoso frecuente en ambas epidermis. Las paredes de las células epidérmicas tienen engrosamientos localizados en las curvas. El tamaño celular varía, el RC difiere en ambas epidermis.

El epifilo (Fig. 2A) está formado por células más largas que anchas, con un RC 2,5:1; paredes con ondas curvas algo engrosadas, frecuentes, amplias y profundas. El hipofilo (Fig. 2B) presenta células irregulares tipo pieza de rompecabeza, de mayor tamaño que las del epifilo, con RC 5:1. Tiene un modelo sinuoso frecuente con ondas cuadrangulares y curvas, profundas casi uniformes, de gran amplitud y levemente engrosadas en las ondas.

\section{Estomas}

En vista superficial, los estomas tienen contorno elíptico y son una vez más largos que anchos. Las células vecinas (Fig. 2B) guardan disposiciones variadas: dos células orientadas perpendicularmente respecto de las oclusivas, estomas diacíticos o, tres células siendo una de ellas más pequeña, estomas anisocíticos. Las dimensiones de los estomas varían de $37(41,18) 50 \mu \mathrm{m}$ de largo $\times 26(28,64) 32,7 \mu \mathrm{m}$ de ancho. Los estomas anisocíticos son el tipo estomático más común.

\section{Rizomas}

Bolbitis serratifolia presenta rizomas de un diámetro aproximado de 5-15 mm, cortamente rastreros y escamosos, que llevan raíces fasciculadas y 2 filas de hojas (Fig. 3E).

\section{Arquitectura foliar}

En B. serratifolia los estípites estériles, distantes entre sí 0,5-1,2 cm, no están 
articulados al rizoma y alcanzan los 25-44,25 (66) cm de long. Son delgados, flexibles, pajizos, escamosos y representan aproximadamente $1 / 3$ de la longitud total de la fronde. Son adaxialmente surcados y abaxialmente lisos. Los raquis son cilíndricos, no alados, surcados en el haz y lisos en el envés, siendo las costas prominentes en el envés.

Las frondes (Fig. 3A) son dimórficas, imparipinnadas, rematan en una pinna apical similar a las laterales pero de mayor tamaño. Las láminas estériles son bicolores, verde oscuro en el epifilo y verde más claro en el hipofilo, presentan contorno elíptico y son prolíferas. El tamaño de las mismas varía entre 50-68,25(77) cm long. $\times 16-32(40) \mathrm{cm}$ de lat. Las láminas llevan 10-22 pares de pinnas distantes 3,5-4(4,5) cm una de otra.

Las pinnas están insertas en el raquis en posición perpendicular ascendente, algo curvadas hacia arriba. Son alternas, papiráceas y no están articuladas al raquis; miden aproximadamente 11-15,92(19) cm long. $\times 2,1$ $2,43(3,2) \mathrm{cm}$ lat. Son lanceoladas, con ápices atenuados, bases asimétricas, oblicuamente excavadas sobre el lado acroscópico y márgenes crenado-aserrado (Fig. 3B). Las pinnas basales presentan bases redondeadas y peciólulos muy breves, que miden entre 1-2 $\mathrm{mm}$, aunque a veces pueden faltar.

La pinna apical mide 13-16,83(19) cm long. $\times 1,5-1,9(2,2) \mathrm{cm}$ lat., y a veces desarrolla 1-2 bulbillos en posición subterminal (Fig. 4A). Los bulbillos (Fig. 4B) son más o menos globulares o esféricos, tienen una estructura persistente, están situados adaxialmente en la pinna y cubiertos con escamas similares a las del rizoma.

Las venas son visibles, del color de la lámina, anastomosadas y delimitan aréolas heteromórficas. Las costas (Fig. 3B) tienen un recorrido recto en todo su trayecto; las venas secundarias, oblicuas y prominentes en sus 2/3 partes, corren paralelas entre sí y forman un ángulo de $60-70^{\circ}$ con la costa, este ángulo de desviación es casi uniforme a lo largo de la pinna. Esparcidas entre las venas secundarias, se encuentran venas intersecundarias o venas terciarias que se anastomosan entre sí formando aréolas (Fig. 3D). Las aréolas están bien desarrolladas (Fig. 4C-D), tienen forma $\mathrm{y}$

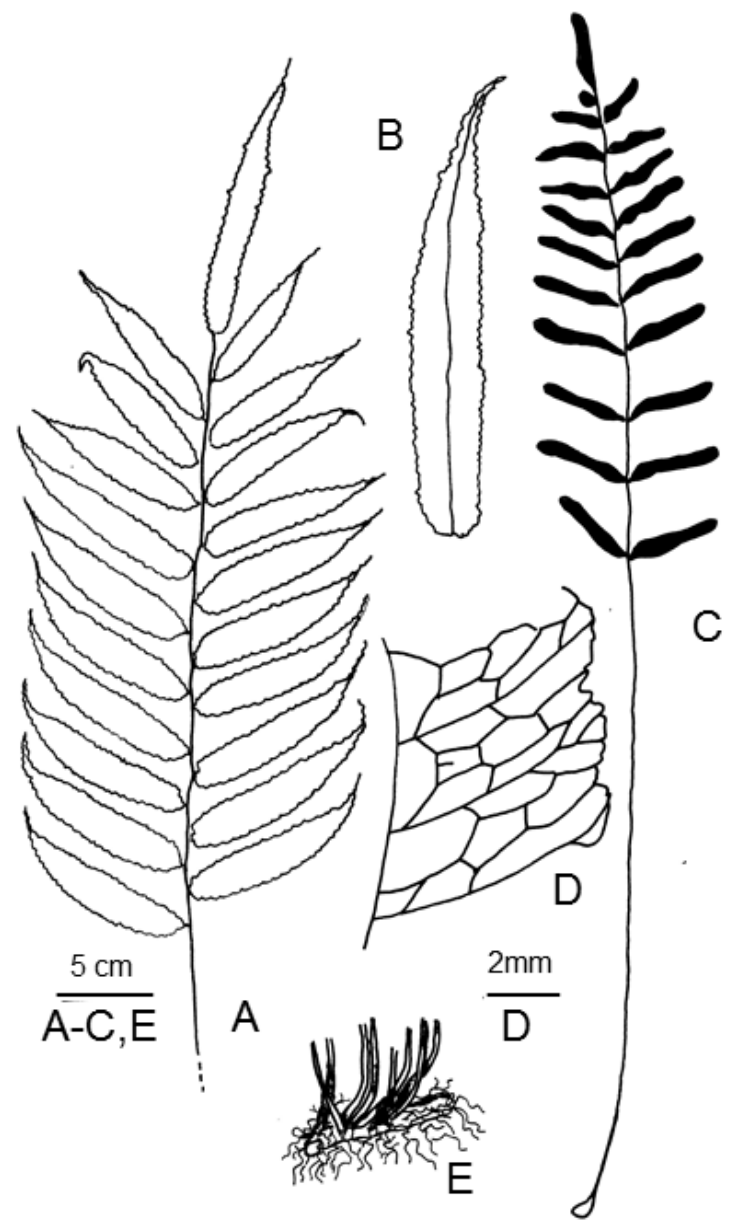

Fig. 3. Bolbitis serratifolia. A: Fronde estéril. B: Pinna estéril. C: Fronde fértil. D: Detalle de la venación. E: Rizoma.

tamaño variable con una disposición al azar. Las aréolas costales son trapezoidales bajas; las distales, pequeñas e irregulares, están distribuidas en 2-3 hileras. La mayoría de las aréolas carecen de venillas y cuando están presentes son libres y excurrentes hacia el margen.

Las frondes fértiles (Fig. 3C) son de menor longitud que las frondes estériles. Los estípites alcanzan los 26-48,41(72) cm long., valores similares a los registrados para los estériles. Las láminas son muy estrechas en comparación con las estériles, miden alrededor de 15$31,16(47) \mathrm{cm}$ long. $\times 5,50-6,50(7,50) \mathrm{cm}$ lat. y llevan 9-16 pares de pinnas distanciadas entre sí por 2-2,50(3,50) $\mathrm{cm}$. Las pinnas 

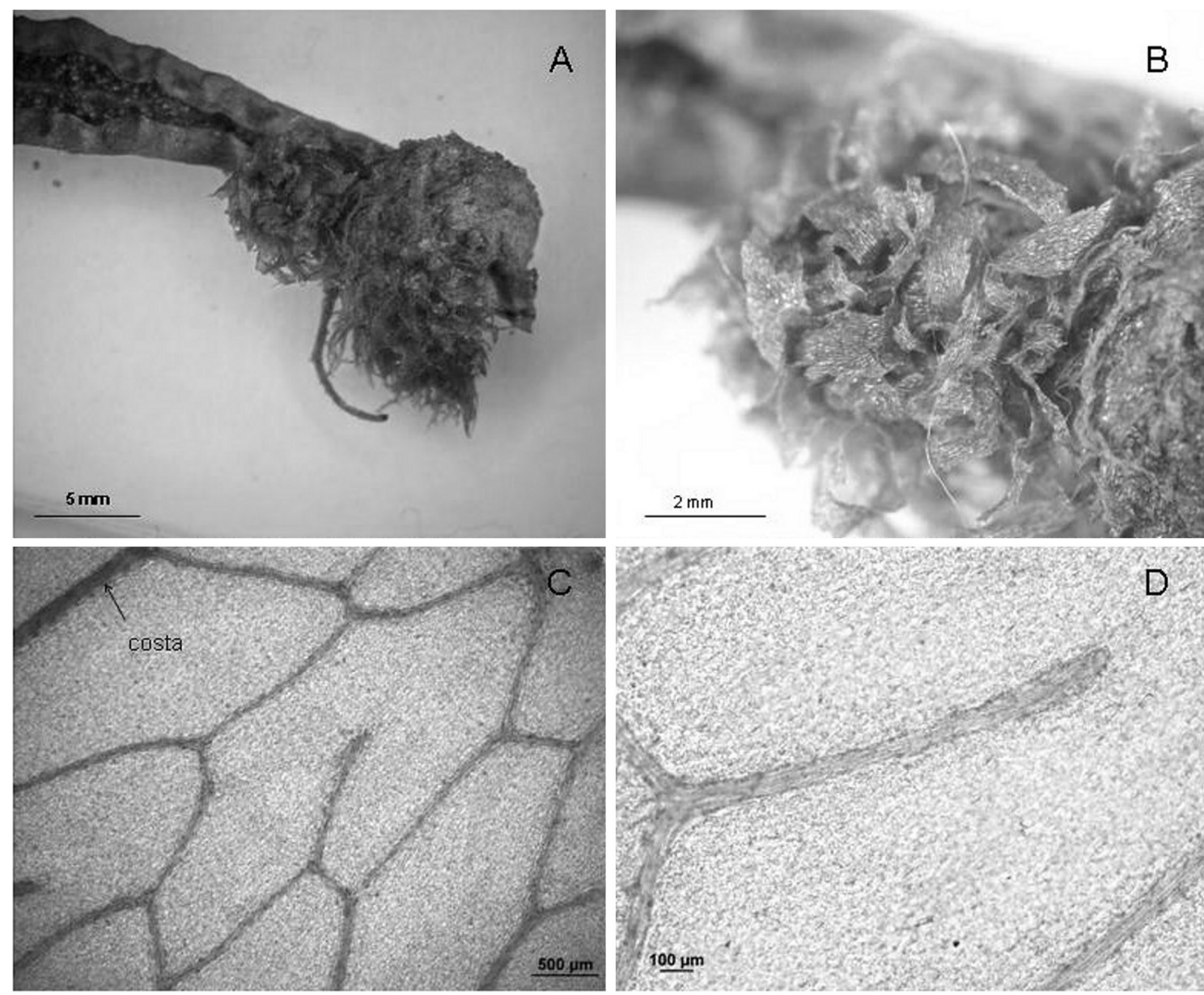

Fig. 4. Bolbitis serratifolia. A: Bulbillo en pinna apical. B: Detalle de bulbillo. C: Venación foliar. D: Detalle de una aréola. (A-B: Capurro 217; C-D: Vervoorst \& Cuezzo 7805 C).

miden aproximadamente $2,5-5,84(10) \mathrm{cm}$ long. $\times 0,6-0,67(0,8) \mathrm{cm}$. lat. Son elípticas, cortamente pecioluladas, con ápices y bases redondeados y márgenes subrevolutos. Están cubiertas en la cara abaxial por esporangios con una disposición acrosticoide, sin indusio ni paráfisis. Según Ranker \& Haufler (2008) en la mayoría de las Dryopteridaceae el indusio ha sufrido una transformación marcada y se ha ido perdiendo independientemente a lo largo de muchas líneas evolutivas.

\section{Esporas}

Las esporas de Bolbitis serratifolia son de forma elíptica a orbiculares en vista polar (Fig. 5A-B) y reniformes en vista ecuatorial (Fig. 5C; Fig. 6H). La forma y el tamaño se mantienen más o menos constantes y no se han encontrado esporas gigantes o aberrantes (Fig. $6 \mathrm{H})$. El diámetro polar es de $38-41 \mathrm{~mm}$ y el ecuatorial de $43-55 \mathrm{~mm}$.

Las esporas son triletes, no se encontraron esporas monoletes ni aletes. La lesura siempre es visible y la ornamentación no la enmascara. Los brazos están generalmente ornamentados, cada uno de ellos alcanza aproximadamente 1/3 del diámetro de la cara proximal (Fig. 5E$\mathrm{G})$.

El esporodermo está formado por un exosporio liso y un perisporio con macro y microornamentación. En el perisporio se distinguen 2 capas: una externa compacta, adpresa, que se desprende con facilidad (Fig. 6A-C), carácter considerado primitivo 

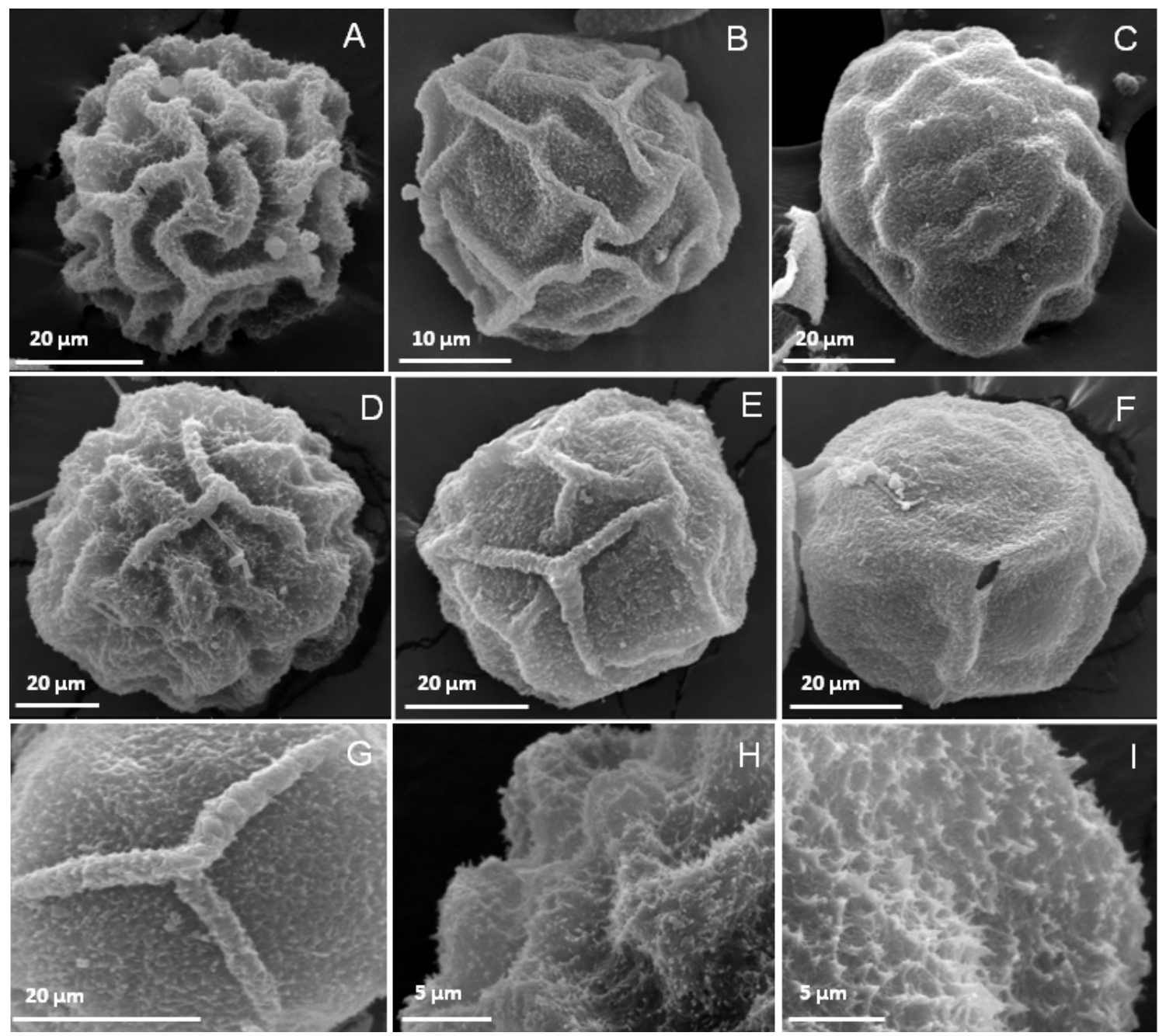

Fig. 5. Esporas de Bolbitis serratifolia con perisporio. A-B: Vista polar distal. C: Vista ecuatorial. D-F: Vista polar proximal. G: Trilete. H-I: Detalle del perisporio. (A, D, H: Schinini 13388; B: Capurro 335; C: Legname \& Cuezzo 8439C; E, G: Capurro 38; F, I: Schulz \& Varela 5179).

por Moran et al. (2010b) y una interna, parcialmente adherida al exosporio, de aspecto laminar casi folioso (Fig. 6A, D, E). E1 perisporio es muriforme, se deposita en forma continua y cubre la lesura (Fig. 5D). La capa más externa es rugada, presenta muros más a menos pronunciados, anchos y redondeados, dispuestos irregularmente, de 2,3-3 $\mathrm{mm}$ de espesor, que ocasionalmente se anastomosan formando aréolas (Fig. 5A-F); presenta microornamentación formada por prolongaciones cónicas, de ápice agudo y superficie granular (Fig. 5H-I). La capa más interna del perisporio es membranosa, retiada, presenta crestas delgadas y aplanadas formando aréolas, con micro-ornamentación espaciada, formada por escamas de contorno irregular y algunos gránulos (Fig. 6D, E, G). El exosporio forma una capa continua, lisa sin microornamentación (Fig. 6F).

\section{Discusión y Conclusiones}

El presente trabajo actualiza la descripción de la especie, estableciendo afinidades y diferencias con otras especies de Dryopteridaceae del noroeste argentino. 

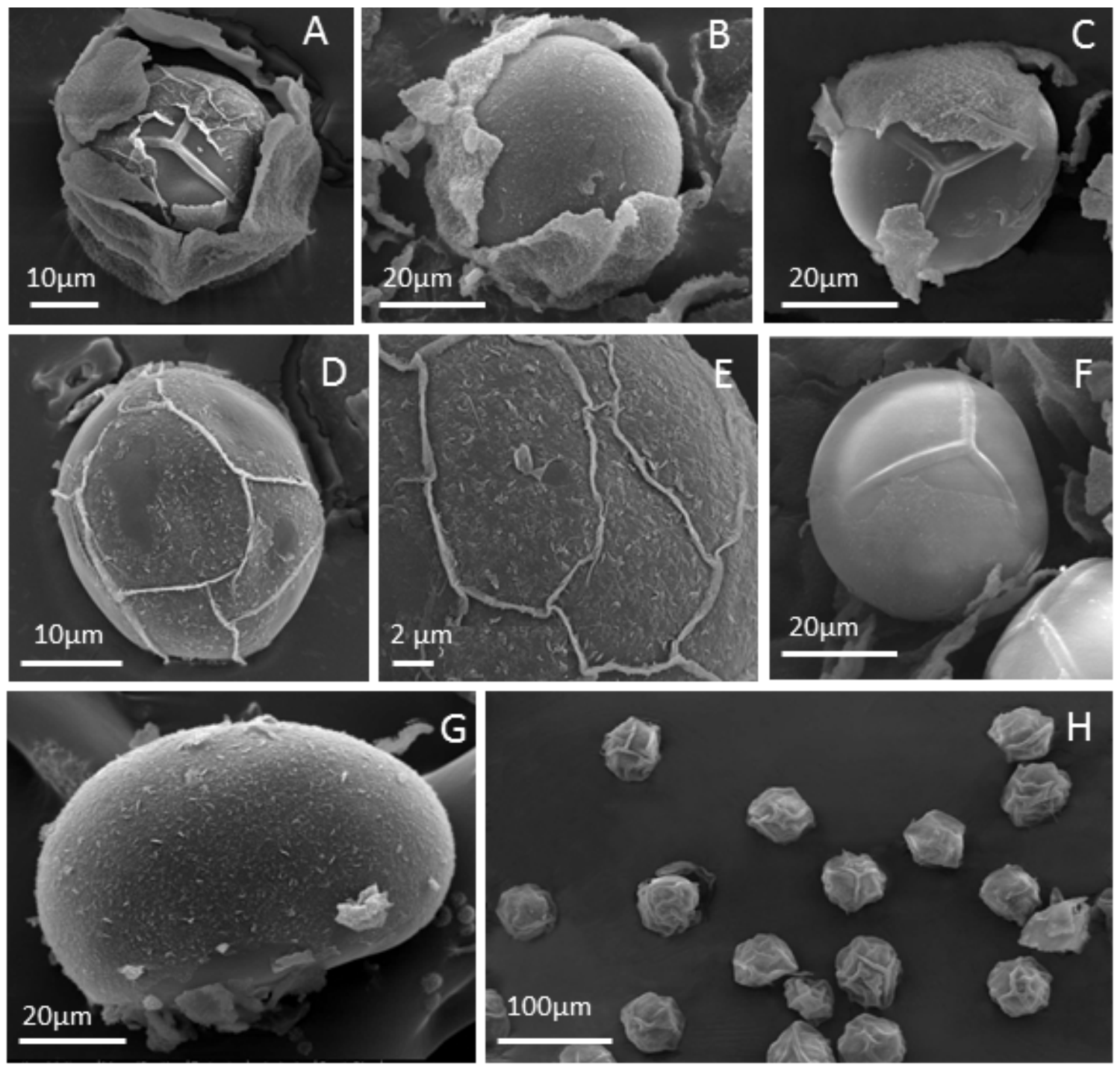

Fig. 6. Esporas de Bolbitis serratifolia. A, C: Exosporio y perisporio en vista polar proximal. B: Exosporio y perisporio en vista polar distal. D, E, G: Perisporio (capa interna). F: Trilete exosporio. H: Vista panorámica. (A, B, D, E, H: Capurro 335; C: Capurro 38; F: Legname \& Cuezzo 8439C; G: Schinini 13388).

La estrecha relación existente entre Bolbitis y Elaphoglossum sugerida por Moran et al. (2007), es corroborada aquí por rasgos morfológicos compartidos por ambos géneros como soros acrosticoides, dimorfismo foliar y un haz vascular ventral alargado en el rizoma.

Otros caracteres del esporófito permiten diferenciar ambos géneros, la presencia de láminas pinnadas y glabras, la venación reticulada y las esporas triletes son distintivas de $B$. serratifolia; mientras que la existencia de láminas simples, subglabras y escamosas, la venación libre y las esporas monoletes caracterizan a las distintas especies argentinas de Elaphoglossum (Lavalle \& Rodríguez, 2009; 2010; 2013).

Bolbitis serratifolia se distingue por presentar ejes escamosos y láminas glabras. El modelo epidérmico de $B$. serratifolia coincide con el de E. piloselloides y E. yungense (Lavalle \& Rodríguez, 2009) sólo se diferencia por la presencia de engrosamientos. 
Los tipos estomáticos parecen ser un rasgo genérico; con predominio de anisocíticos en $B$. serratifolia y de diacíticos y anomocíticos en Elaphoglossum.

Las esporas evidencian una abertura trilete, esto difiere con lo aportado por otros autores (Kaur, 1974; de la Sota, 1977; Hennipman, 1977; Kramer, 1990; Tryon \& Lugardon, 1991; Moran, 1995). Las autoras coinciden con Moran et al. (2010b) en la presencia de un perisporio suelto, no adpreso, con anchos pliegues y microornamentación, característico de los helechos bolbitidioides.

El indumento, los modelos epidérmicos y las esporas son rasgos diagnósticos específicos que ya han sido puesto a prueba en las siete especies argentinas de Elaphoglossum (Lavalle \& Rodríguez, 2010; 2013).

\section{Bibliografía}

CHRISTENHUSZ, J. M.; X. ZHANG \& H. SCHNEIDER. 2011. A linear sequence of extant families and genera of lycophytes and ferns. Phytotaxa 19: 7-54.

HENNIPMAN, E. 1977. A monograph of the fern genus Bolbitis (Lomariopsidaceae). Leiden Botanical Series 2: 171-175.

HOLMGREN, P. K., N. H. HOLMGREN \& L. BARNETT. 1990. Index Herbariorum: Herbaria of the World, Part I. New York Botanical Garden, Bronx, New York.

KAUR, S. 1974. The family Lomariopsidaceae (Filicopsida) and its probable ancestors. J. Linn. Soc., Bot. 68: 153-162.

KRAMER, K. U. 1990. Lomariopsidaceae. En: K. Kramer \& P. S. Green (eds.), The families and genera of Vascular Plants I, Pteridophytes and Gymnosperms, pp. 164-170. Springer-Verlag, Berlin.

LAVALLE, M. C. \& M. RODRÍGUEZ. 2009. Taxonomía de las especies argentinas de Elaphoglossum (Dryopteridaceae - Pteridophyta). Darwiniana 47: 125-139.

LAVALLE, M. C. \& M. RODRÍGUEZ. 2010. Indumento y modelos epidérmicos en especies argentinas de Elaphoglossum (Dryopteridaceae - Pteridophyta). Bol. Soc. Argent. Bot. 45: 245-256.

LELLINGER, D. B. 2002. A Modern Multilingual Glossary of Taxonomic Pteridology. Pteridologia 2A: 1-364.

LI, C. X. \& C. G. LU. 2006. Phylogenetic analysis of Dryopteridaceae based on chloroplast $r b c \mathrm{~L}$ sequences. Acta Phytotax. Sin. 44: 503-515.
MICKEL, J. T. 1991. Elaphoglossum. En: R. TRYON \& R. G. STOLZE (eds.), Pteridophyta of Peru, Part IV, 17. Dryopteridaceae. Fieldiana, Bot. n. s. 27: 98-165.

MICKEL, J. T. 1999. Elaphoglossum. En: P. BERRY; B. HOLST \& K. YATSKIEVYCH (eds.), Flora of the Venezuelan Guayana, vol. 2, pp. 89-101. Missouri Botanical Garden Press, St. Louis.

MICKEL, J. T. \& A. R. SMITH. 2004. The Pteridophytes of Mexico. Mem. New York Bot. Gard. 88: 282-315.

MORAN, R. C. 1995. Lomariopsidaceae. En: G. DAVIDSE; M. SOUZA \& S. KNAPP (eds.), Flora Mesoamericana I, Psilotaceae a Salviniaceae, pp. 247-282. Universidad Nacional Autónoma de México, México, D. F.

MORAN, R. C.; J. GARRISON HANKS \& G. ROUHAN. 2007. Spore morphology in relation to phylogeny in the fern genus Elaphoglossum (Dryopteridaceae). Int. J. Plant Sci. 168: 905-929.

MORAN, R.; M. SUNDUE \& P. LABIAK. 2010a. Phylogeny and character evolution of the Bolbitidoid ferns (Dryopteridaceae). Int. J. Plant Sci. 175: $547-$ 559.

MORAN, R.; J. GARRISON HANKS; P. LABIAK \& M. SUNDUE. 2010b. Perispore morphology of Bolbitidoid ferns (Dryopteridaceae) in relation to phylogeny. Int. J. Plant Sci. 171: 872-881.

PICHI SERMOLLI, R. E. G. 1969. Adumbratio Fl. Aethiopica, 18. Lomariopsidaceae. Webbia 23: 379-396.

PONCE, M. M. 1996. Pteridophyta. En: F. ZULOAGA \& O. MORRONE (eds.), Catálogo de las Plantas Vasculares de la República Argentina I. Monogr. Syst. Bot. Missouri Bot. Gard. 60: 29-31.

PONCE, M. M. \& E. R. de la SOTA. 2008. Lomariopsidaceae. Pteridophyta. En: F. ZULOAGA, O. MORRONE \& M. BELGRANO (eds.), Catálogo de las Plantas Vasculares del Cono Sur (Argentina, sur de Brasil, Chile, Paraguay y Uruguay). Monogr. Syst. Bot. Missouri Bot. Gard. 107: 76-78.

PRABHAKAR, M. 2004. Structure, delimitation, nomenclature and classification of stomata. Acta Bot. Sin. 46: 242-252.

RANKER, T. A. \& C. H. HAUFLER. 2008. Biology and Evolution of Ferns and Lycophytes. Cambridge University Press, Cambridge UK. 480 pp.

ROLLERI, C. \& A. DEFERRARI. 1986. Modelos epidérmicos y otros caracteres foliares en la sistemática y ecología de especies de Lycopodium L. Sección Crassistachys Herter. Revista Mus. La Plata, n.s., Bot. 14: 65-87.

SMITH, A. R. 1981. Pteridophytes. En: D. E. BREEDLOVE (ed.), Flora de Chiapas, vol. 2. pp. 61-64. California Academy of Sciences, San Francisco.

SMITH, A. R., K. M. PRYER, E. SCHUETTPELZ, P. KORALL, H. SCHNEIDER \& P. G. WOLF. 2006. 
A classification for extant ferns. Taxon 55: 705-731. SOTA, E. R. DE LA. 1973. Sinopsis de las Pteridofitas del Noroeste de Argentina, II. Darwiniana 18: 240-248. . 1977. Elaphoglossum. En: A. L. Cabrera (ed.), Flora de la Provincia de Jujuy. Colecc. Ci. Inst. Nac. Tecnol. Agropecu. 13: 230-240.
TRYON, A. F. y B. LUGARDON 1991. Spores of the Pteridophyta, pp. 514-515. Springer Verlag, New York.

TRYON, R \& A. TRYON. 1982. Ferns and allied plants with special reference to tropical America. pp. 599607. Springer-Verlag, New York-Berlín.

Original recibido el 9 de octubre de 2013; aceptado el 18 de noviembre de 2013. 